\title{
DISCUSSION (Hensberge and van Rensbergen)
}

KHOKHLOVA: It is difficult to distinguish between genuine spectroscopic binaries and stars with spotty distributions of metals, which show velocity variations as they rotate. What precautions were taken to minimize this problem, especially for $S i$ and SrCrEu stars? A good example is the case of $\varepsilon$ UMa, which for a long time was listed as a binary, until careful investigation revealed that the variations were due to the spotted distribution of elements.

HENSBERGE: If I recall correctly, variations from metal lines were rejected and only data from hydrogen Balmer lines, and other lines like $\mathrm{Mg}$ II $4481 \mathrm{~A}$ (which tend not to be variable in these stars) were used. The percentage of binaries was determined by counting stars which had either a variable radial velocity or were mentioned as spectroscopic binaries, in a sample restricted to the Bright Star Catalogue. Suspected binaries or velocity variables were not taken into account. The numbers I quote may be in this sense upper limits.

KHOKHLOVA: How large was the sample for these statistics?

HENSBERGE: The sample contained $31 \mathrm{He}$-weak stars, $120 \mathrm{Si}$ stars, $97 \mathrm{Hg}-$ Mn stars and 113 cool CP2 stars.

KHOKHLOVA: Good, that is sufficient. Recently, Kopylov and Klochkova (Special Astrophysical Observatory) made an interesting investigation of stars in clusters.

HENSBERGE: I found a reference but I did not have time to trace the paper.

DROBYSHEVSKI: I wish to present some additional information concerning the duplicity of Am stars. This was done several years ago (E. M. Drobyshevski, Genesis and Classification of the Magnetic Stars II. Am Stars and Binarity of Early Stars, Preprint PhTI-445, Leningrad, 1973) but was not published for a wide distribution. I repeated $G$. P. Kuiper's analysis which was made for only 26 SB1 MS systems with orbits known at the time (Publs. Astron. Soc. Pacific, 47, p. 15, 1935). For random orbit orientations, he obtained a relation between the observed distribution $\phi(\nu)$ and the true distribution $\psi(\nu)$

$$
\phi(\nu)=\nu \int_{\nu}^{\nu} \psi(\mu)\left(\mu^{2}-\nu^{2}\right)^{-1 / 2} \mu^{-1} d \mu
$$

where $\mu=M_{1} /\left(M_{1}+M_{2}\right)$ and $\nu=\mu \sin i=\left[f(M)\left(M_{1}+M_{2}\right)^{-1}\right]^{1 / 3} ; f(M)$ is the mass function.

As data $I$ used the second catalogue of SB systems (A. H. Batten, Publs. Domin. Astr. Obs., 13, p. 119, 1967) which contains data on 39 SB1 and SB2 systems with Am-type components and on 27 systems with normal A components. Only bright systems with $2 \mathrm{~d} \leq \mathrm{P}_{\text {orb }} \leq 200 \mathrm{~d}$ and quality $a, b$, c were used.

In Figs. 1a and 1b, distributions $\phi(\nu)$ for Am and A systems are presented. Also shown are distributions $\psi(\mu)$ and $\psi^{\prime}(\mu)$ obtained by means of integration of the distribution. When obtaining $\psi^{\prime}(\mu)$, the 163

C. R. Cowley et al (eds.), Upper Main Sequence Stars with Anomalous Abundances, 163-166.

(C) 1986 by D. Reidel Publishing Company. 
observational selection was taken into account by assuming the $S B$ detection probability to be proportional to $v^{-1}$.

From these diagrams, one can see that Am SB systems separate into two groups: (1) $q=M_{2} / M_{1}>0.5$, and (2) $q<0.4$. It is only natural to assume the first systems to be young, and the second group evolved, with invisible secondary components (white dwarf type?).
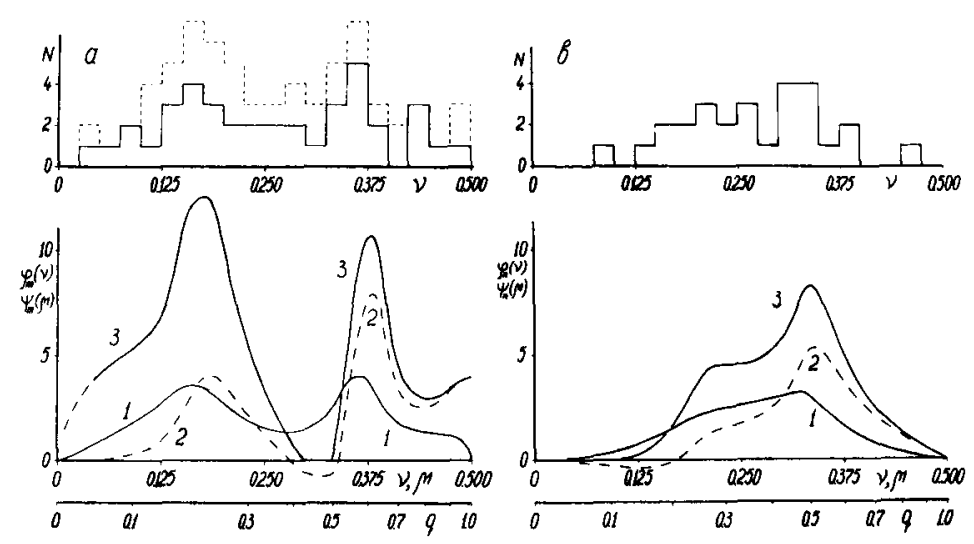

$$
\text { Fig } 1 .
$$

Distribution for SB systems with Am (a) and normal A (b) components. The dashed line is a histogram for all the Am SB systems known (including faint systems, those of any period, and low quality orbits). Curve (1) is the $\phi(\nu),(2)$ is the $\psi(\mu)$, and (3) is the $\psi^{\prime}(\mu)$ distribution, which takes into account observational selection.

The number of Am systems in group (2) is twice as great as the number in group (1). Such a result explains why Am stars are observed both in very young, and older, clusters.

To some extent, the distribution of systems with "normal" A star components fills the gap between the young and old Am systems. Nevertheless, for A stars as a whole, the bimodal distribution is conserved, as was found by M. Trimble, and by Kraicheva, Tutukov and Yungelson. In its turn, the number of Am systems is twice that of the normal A systems.

In 1973, when this work was done, only 223 bright Am stars were known, among them $92 \mathrm{SB}(41 \%)$ including 62 systems with known orbits (7 eclipsing, $17 \mathrm{SB} 2$ and $41 \mathrm{SB} 1)$. On the other hand, it follows from comparison of

$$
\int_{0}^{0.5} \psi_{m}^{\prime}(\mu) d \mu \quad \text { with } \quad \int_{0}^{0.5} \phi_{m}(v) d v
$$

that only $40 \%$ of all SB stars with Am components are detected. From the coincidence of these figures $(41 \%$ and $40 \%)$ one may conclude that all the 
Am stars are binaries.

STEPIEN': I wonder what meaning we can assign to the 'average' radius of an Ap star. These stars are found over a wide range of spectral types, from about $B 4$ or $B 5$, to around $F 2$, which means that the range of radii would be expected to be a factor of, well, several. Would it not be more reasonable to restrict an analysis to a narrower spectral interval, say $\mathrm{B} 8$ to $\mathrm{A} 2$, and to compare an average from this interval with the radii of normal stars?

HENSBERCE: I agree with you. The best solution would be to derive for a well-defined sample of CP stars the distribution of the radii. With more photometric periods becoming available, and plausible assumptions about the distribution of the inclination angle of rotation axes with respect to the observer, this could be attempted using the period and Iine-width data.

However, only a few stars have been studied with the infrared flux method or by using the Barnes-Evans relation, perhaps only about ten stars. This means that definition of the subgroups is not possible. I quoted mean values here to show that systematic differences in results, from different methods and samples, do not amount to more than $20 \%$. I emphasize with you that the use of an average radius has no physical meaning.

What you propose might be possible for the data assembled by Babu and Shylaja, because that sample contains about 80 to 90 CP stars, I think.

SCHÖNEICH: It is very important, for the theory of their origin, that the frequency of the magnetic CP stars in binaries is now higher. Previously, it was thought that this frequency was about 20-25\%; now it is nearly normal. The former numbers could be interpreted as an absence of short periods and of circular orbits, but now we must assume that the orbits are changed, because the number of systems is the same as for normal A stars.

HENSBERG: The comparison may be less straightforward than it seems, if one takes into account that the distribution of mass functions for CP and normal stars could be different. Indications for such a difference for Am stars was just described by Drobyshevski, who claims a bimodal distribution for $f(m)$.

KOPYLOV: I would like to make two comments on the last part of this survey. First, it is necessary to take into account the individual properties of every cluster which contains CP stars; there are, for example, both young and old clusters which contain them. Second, we have examined CP stars in open clusters by quantitative spectroscopic methods (108 stars in 10 clusters). We find that there is no real change of $v$ sin $i$, quantitative spectral peculiarity index, or $\left\langle B_{e}\right\rangle$ during the evolution of CP stars across the main sequence band. 


\section{DISCUSSION (Klochkova and Kopylov)}

COWLEY: I'd like to make a comment on the weakness of the helium lines, which in the simplest interpretation reflects a lower He abundance. If the normal comparison stars have similar colours, then we all know that the He lines will be weaker in the peculiar stars. But, if you choose stars of the same effective temperature, we must consider other factors, because we know that the ground-based colours lead to a different effective temperature when the chemistry of the star is unusual. The effects of line blanketing suggest that we should use cooler normal stars for comparison purposes, and this should reduce the anomaly in the He line strengths.

KOPYLOV: Yes. This problem is well known to us. But, note that the abscissa in our plots (such as $W$ vs. $S p$ ) is the effective temperature rather than the spectral type proper. For every star in our sample it was possible to find the effective temperature by the ionization equilibrium method and to compare this with the value obtained from space observations. For this reason, we think that the effective temperature scales for $\mathrm{CP}$ and normal stars do not differ significantly, and so the weakness of He is real, and not some mistake or error in the analysis.

DROBYSHEVSKI: From your data on the absence of a correlation between the peculiarity index and the age, it follows that the diffusion theory of chemicaliy peculiar stars must be questioned. What can you say on this subject?

KOPYLOV: Our recent results are in some disagreement with the diffusion hypothesis. We are not surprised at this, because this theory is still being developed. Because many results for CP stars can be explained at present by this theory, we can express the hope that future modifications could lead to explanations for the main conclusions we have made in our statistical study. We see no real alternative to this theory for the explanation of the variety of individual properties of $C P$ stars. This is a problem for the future.

MICHAUD: We could discuss that this afternoon, but I do not think there is any contradiction, because the time scales to establish the anomalies once the star arrives on the main sequence is very short. 